\title{
Predicting Noninfectious Pulmonary Complications in HIV Positive Patients based on Persistence of the HIV-Nef Protein
}

Christian Arce Guzman ${ }^{1}$, Sarvesh Chelvanambi ${ }^{2,3}$, Homer L. Twigg III2, Matthias Clauss ${ }^{2}$

${ }^{1}$ Indiana University School of Medicine, ${ }^{2}$ Indiana University School of Medicine, Division of Pulmonary, Critical Care, Sleep and Occupational Medicine, ${ }^{3}$ Department of Medicine, Brigham and Women's Hospital

Background and Hypothesis: According to the Veterans Aging Cohort Study, people living with HIV (PLWH) in the era of antiretroviral therapy (ART) have incidence rates of COPD, pulmonary hypertension and pulmonary fibrosis that are 16,50 and $50 \%$ higher than the general population, respectively. Despite effective treatment, the HIV-Nef protein continues to persist in the blood and bronchoalveolar lavage (BAL) fluid of HIV positive patients. Studies have shown that HIV-Nef promotes inflammation and induces apoptosis within pulmonary immune and endothelial cells, respectively. HIV-Nef transgenic mice display pulmonary disease phenotypes including reduced number of alveoli, increased inspiratory capacity and hypoxemia. Nef persistence in lungs could explain the high rates of pulmonary complications in PLWH. We hypothesize that patients with HIV-Nef are more likely to exhibit pulmonary dysfunction.

Methods: A secondary data analysis was conducted on samples collected from 34 HIV positive patients with variable HIV-Nef levels in plasma and BAL fluids as detected by ELISA. Using GraphPad Prism 8 and $p<0.05$ for statistical significance, bivariate analyses were performed, including Spearman correlation, simple linear regression and Welch's $t$ test.

Results: In patients with no detectable HIV-Nef in BAL fluids, pulmonary function test (PFT) parameters, such as FEV1/FVC, FEV1 and FEF 25-75 showed a positive correlation with CD4 counts; however, preservation of CD4 counts did not normalize PFT measures in Nef positive patients. HIV-Nef presence in the lungs resulted in higher levels of chemokines and proinflammatory cytokines, which led to increased percentages of infiltrating lymphocytes and neutrophils.

Conclusion and Potential Impact: Our findings suggest that HIV-Nef mediates inflammation in the lungs and disrupts the protective effect of preserving CD4 count on pulmonary function. Screening for HIV-Nef could become a routine test to predict risk of pulmonary complications. Therapies that target Nef induced pulmonary inflammation could reduce the prevalence of pulmonary diseases in PLWH. 